\title{
Potential role of PET/MRI in radiotherapy treatment planning
}

\author{
Daniela Thorwarth $\cdot$ Sara Leibfarth \\ David Mönnich
}

Received: 26 October 2012 / Accepted: 19 January 2013/Published online: 16 February 2013

(C) Italian Association of Nuclear Medicine and Molecular Imaging 2013

\begin{abstract}
State-of-the art radiotherapy (RT) techniques have become extremely flexible. As a consequence, highly conformal target doses in combination with optimal organat-risk sparing are possible. Due to this highly precise application of complex dose distributions, treatments with higher radiation doses in the tumor but without increased toxicity levels are now feasible. This offers the possibility to adapt dose distributions according to individual radiation sensitivities as measured with functional imaging modalities. Recently, combined PET/MRI has become available. This novel hybrid imaging modality offers the possibility to simultaneously image anatomical, functional and molecular characteristics of a tumor. Consequently, PET/ MRI may be an optimal basis for RT individualization. This review discusses different potential applications of PET/MRI for RT treatment planning, as well as technical challenges for the integration of multi-parametric PET/ MRI data into RT treatment planning.
\end{abstract}

Keywords Combined PET/MRI - Radiotherapy - Dose painting $\cdot$ Multi-parametric imaging

\section{Introduction}

Technologically highly advanced techniques, such as intensity-modulated radiotherapy (IMRT) [35], volumetric modulated arc therapy [38], and proton or particle therapy

\footnotetext{
D. Thorwarth $(\varangle) \cdot$ S. Leibfarth · D. Mönnich

Section for Biomedical Physics, Department of Radiation Oncology, University Hospital Tübingen, Eberhard Karls University Tübingen, Hoppe-Seyler-Str. 3, 72076 Tübingen, Germany

e-mail: daniela.thorwarth@med.uni-tuebingen.de
}

[37], have made modern radiotherapy (RT) treatments extremely flexible in terms of shaping the delivered target doses. As a consequence, conformal radiation doses can be delivered to the tumor volume and, at the same time, optimal sparing of the organs at risk and surrounding normal tissue can be achieved [14]. Reliable application of these complex techniques on a day-to-day basis can be guaranteed by imaging the patient directly in the treatment room with e.g., cone-beam computed tomography [43]. Also, techniques that allow the irradiation of moving targets such as lung tumors are currently being introduced into clinical RT practice [43]. By combining highly flexible RT hardware with sophisticated treatment planning software, it is now possible to increase the dose inside the tumor without increasing the toxicity levels [3, 51].

Functional and molecular imaging techniques using magnetic resonance imaging (MRI) or positron emission tomography (PET) allow visualization of various pathophysiological properties of tumors which may be of interest for assessing individual parameters related to accurate diagnosis or radiation sensitivity $[36,55]$. Consequently, Ling et al. proposed dose painting to increase the radiation dose to a biological target volume, which consists of a subvolume of the planning target volume (PTV), delineated on the basis of functional imaging information [28]. A further refinement of this approach is the possibility to shape the dose gradually according to the functional parameter values using dose painting by numbers [3,5]. Since the idea of dose painting was first published, several planning studies have been performed that confirmed the technical feasibility of shaping the radiation dose according to functional characteristics determined by functional and molecular imaging with PET or MRI [3, 31, 51].

In addition to PET/CT and MRI, a new hybrid imaging technology, PET/MRI, has recently entered clinical practice 
[25, 40]. Combined PET/MRI allows the simultaneous acquisition of PET and MRI images, and thus, might offer great opportunities for cancer research and therapy [13, 45, 58]. To date, different hardware concepts that combine PET and MRI have been proposed and realized by different vendors [19]. Basically, three different categories of clinical PET/MRI hardware can be distinguished: (1) separate gantries in different rooms [56], (2) co-planar concepts where scanner gantries are in the same room and connected by a patient handling system, i.e., patient couch on rails [19], and (3) fully integrated systems [57]. In 2006, a first prototype system was presented which consisted of a magnetic resonance-compatible PET detector ring that could be inserted into a clinical 3 Tesla MR scanner. This prototype system allowed brain imaging only [8, 46]. Realizing an integrated design is extremely challenging, as the PET detector system has to be modified to operate in the magnetic field of the MR scanner [57]. In the meantime, integrated whole-body PET/ MRI systems have also become available (mMR, Siemens Healthcare). A recent study investigated the performance of the PET component of the Siemens mMR [11] and showed results comparable to those obtained with state-of-the-art PET/CT systems [23]. A major technical challenge of integrated PET/MRI systems is the PET attenuation correction [21]. While for combined PET/CT, attenuation correction of the PET emission data is performed using a transformation of the readily available CT images [26], no such correction maps are available for PET/MRI. To overcome this lack of information, different MR-based attenuation correction (MR-AC) methods have been proposed. First, clinical PET/MRI systems use segmentation-based approaches for MR-AC, which have been shown to work well $[6,33]$. More complex strategies, such as the generation of pseudo-CT values using deformable image registration algorithms in combination with pattern recognition, have also been developed [20]. A recent study compared segmentation-based MR-AC to a strategy using atlas registration and pattern recognition. The latter was found to provide a more accurate quantitative PET reconstruction [22]. Nevertheless, MR-AC is a challenging issue which is affected by a variety of different factors, such as the use of MR contrast agents, flexible MR body coils and motion or metal artifacts [10, 30].

This review provides an overview of different PET/MRI techniques and imaging possibilities, focusing in particular on the potential role of PET/MRI for RT treatment planning in the future.

\section{PET/MR imaging for radiotherapy treatment planning}

A main aim of integrating anatomical, functional and molecular imaging into RT planning is to delineate the target volume as precisely as possible. The requirements, in terms of image quality, needed to achieve this goal might differ considerably from those of diagnostic examinations. For RT target volume delineation, high, ideally isotropic, resolution of functional images in combination with high geometric precision is essential.

Hybrid PET/MRI offers intrinsic co-registration of anatomical or functional MRI with molecular PET information. This hardware alignment is highly beneficial in terms of accurate target volume delineation, as errors introduced by subsequent image co-registration are minimized. However, a suitable method for the co-registration of planning CT and MR image volumes is essential. Furthermore, combined PET/MRI provides logistical benefits. For example, in patients requiring RT planning CT, MRI and PET, the total number of RT-specific examinations is reduced from three to two.

Anatomical imaging such as T2-weighted MRI provides information about tumor spread. Nevertheless, functional imaging methods such as $\left[{ }^{18} \mathrm{~F}\right]$-fluorodesoxyglucose (FDG) PET are much more effective for assessing the extension of the tumor mass in some entities [47]. FDG is the most frequently used PET marker in clinical practice today. FDG PET provides information about the metabolic activity of the tumor and hence, is strongly related to the cell density in this region. Studies have shown its ability to provide detailed information about tumor extent, cell density and the prognostic value of this imaging modality for RT outcome in different entities [1, 2]. Potentially, FDG will, in the future, be a key tracer in PET/MRI applications for different clinical purposes. On the one hand, FDG PET/ MRI allows highly accurate definition of tumor volumes based on the molecular PET and the anatomical and functional MRI components. In addition, especially in head and neck cancer (HNC), FDG PET/MRI might offer possibilities to determine probability-based measures for the presence of tumor, a prospect that appears very attractive for adapted RT strategies.

Other tracers such as $\left[{ }^{11} \mathrm{C}\right]$-methionine and $\left[{ }^{68} \mathrm{Ga}\right]-$ DOTATOC have also been shown to provide reliable information about the geometric tumor extension in meningioma $[4,16]$. In prostate tumors, the use of $\left[{ }^{11} \mathrm{C}\right]-$ choline (CHO) for assessing the main tumor burden is still under discussion [54]. Nevertheless, CHO PET/MRI might be of added value for target volume delineation of primary and recurrent prostate cancer, as well as in identifying prostate cancer lymph node involvement [41].

First results show that for accurate contouring of meningioma, combined $\left[{ }^{68} \mathrm{Ga}\right]-D O T A T O C$ PET/MRI can improve the quality of target volume delineation and may, thus, have direct consequences for high-precision RT treatment planning [52] (Fig. 1).

Also, functional MRI techniques allow visualization of structural or pathophysiological features of tumor tissue. 

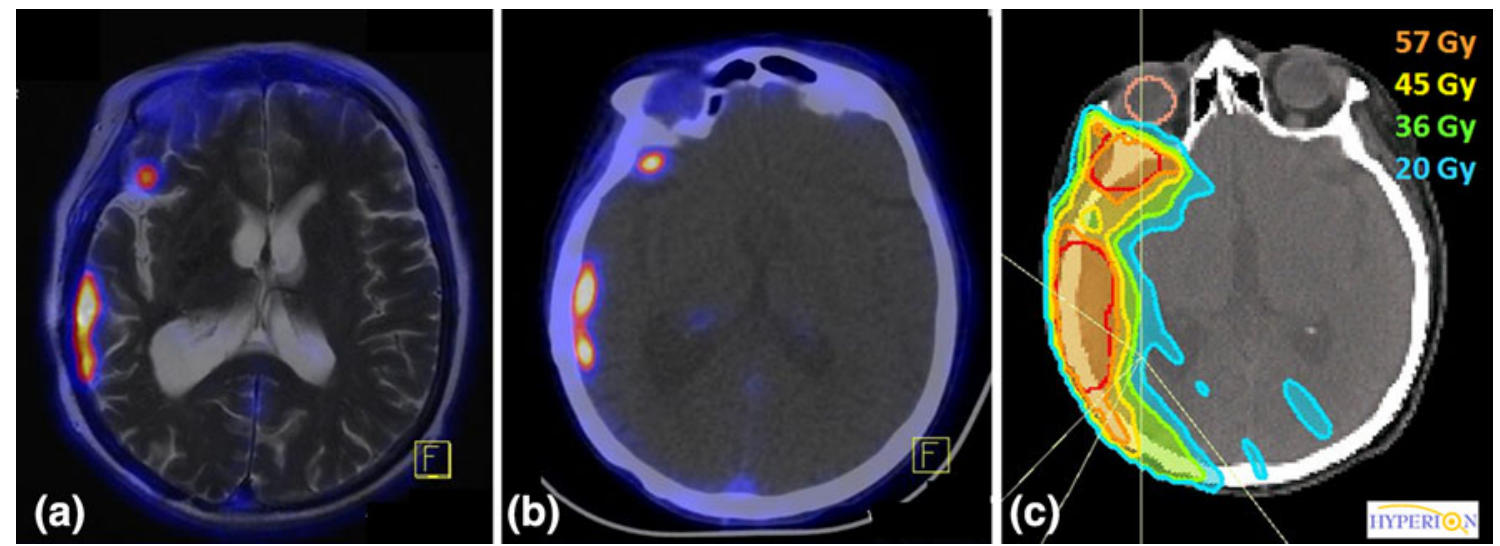

Fig. 1 Combined $\left[{ }^{68} \mathrm{Ga}\right]$-DOTATOC PET/MRI (a) and corresponding PET/CT (b) of a 67-year-old meningioma patient. c IMRT treatment plan, where T2-MRI information as well as DOTATOC uptake were used to create the gross target volume (GTV). PET/MRI,

Diffusion-weighted (DW) MRI visualizes the mobility of water molecules in the tissue. Thus, DW MRI images the 'microanatomy' of the tumor [55], which is characterized by the cell density and by the organization of the cellular and extracellular space. In general, tumors show a reduced diffusion, which is mainly due to the increased cell density, but there are also other influencing factors [39]. Dynamic contrast-enhanced (DCE) MRI consists of a temporal sequence of images that monitor the influx and distribution of a contrast agent in vessels and tissue [9]. DCE-MRI allows the examination of tumor vascularization. Recent studies have shown that DCE and DW MRI may provide important information on early treatment response [44].

For improved RT treatment planning, the availability of simultaneously acquired PET/MRI data may be more valuable than the availability of combined PET/CT information. In particular, when it is necessary to have the highest possible precision in terms of target volume delineation, as for example in meningioma and other brain tumors, PET/MRI may be highly beneficial. Furthermore, high quality of biological multi-parametric imaging combining PET and MRI measures, which may in the near future be relevant for adapted RT treatments of HNC, can only be acquired using PET/MRI (Fig. 2).

\section{Multi-parametric imaging}

One of the main advantages of combined PET/MRI is the possibility of performing high-level multi-parametric imaging in a simultaneous acquisition setting. As a consequence, different pathophysiological measures, such as vascularization, metabolism, perfusion or hypoxia, can be visualized simultaneously on a voxel basis. The integration of multi-parametric functional imaging data into RT
PET/CT and planning CT were registered using a rigid image fusion algorithm which is based on mutual information. Planning target volume (PTV, red contour) was prescribed 60 Gy (color figure online)

treatment planning offers great opportunities for personalized RT strategies in the future.

A variety of studies have highlighted the extreme complexity, in many different regards, of tumor biology. Therefore, to image the main factors of treatment resistance, it may be necessary to apply multi-parametric imaging sequences in a clinical setting.

The literature contains different studies that investigated the potential of multi-parametric imaging in RT [12, 17, 24] and concluded that the potential for integrating multiparametric imaging into RT planning may be considerable. Fig. 3 shows an example where multiparametric PET/MRI data (CHOPET, T2-weighted MRI, DCE- and DW-MRI) have been used to identifiy the primary lesion for a dose escalated treatment in prostate cancer. In the case of prostate cancer, Groenendaal et al. [17] recently developed and validated a multi-parametric model based on DW- and DCE-MRI for delineating prostate cancer. The model even allows the definition of certain risk levels of tumor presence and may therefore be a robust input for dose painting. Another study by Turkbey et al. [53] showed that T2weighted MRI, DCE-MRI and MR spectroscopy provided independent, additive information for prostate tumor detection. In the Netherlands and Belgium, a first clinical trial has been started in which multi-parametric MRI is used for dose painting in prostate cancer [29].

Research in the field of functional imaging in $\mathrm{HNC}$, but also in other tumor entities, focuses mainly on the detection of tumor hypoxia. Tumor hypoxia is one of the main reasons for increased radiation resistance. As a consequence, non-invasive detection of the tumor hypoxia could be extremely beneficial in terms of individual RT adaptation. Functional imaging of hypoxia could provide a direct measure of individual radiation sensitivity and thus, be a powerful input for dose painting. One method of assessing 

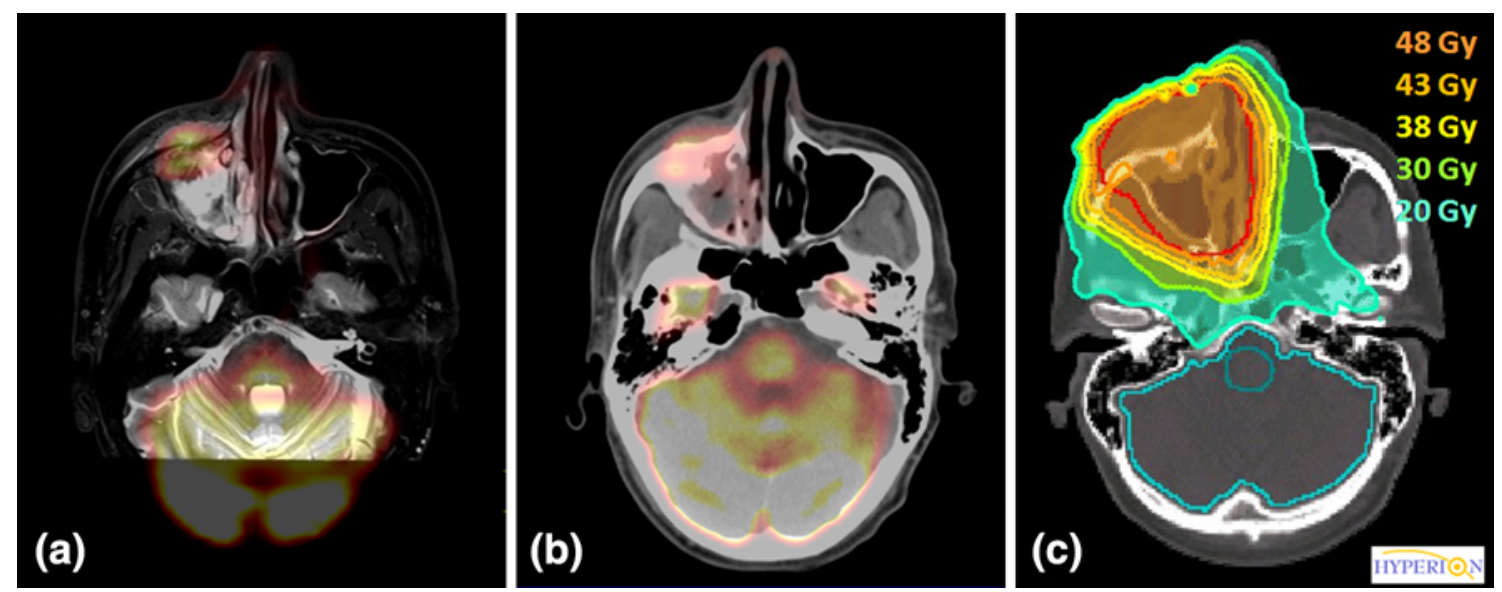

Fig. 2 Simultaneous $\left[{ }^{18}\right.$ F $]$-FDG PET/MRI of a 41-year-old patient with sinus cancer (a) and corresponding PET/CT (b). GTV was contoured on both T2-STIR MRI and FDG PET data. IMRT plan with

the tumor hypoxia non-invasively is by means of PET using dedicated hypoxia tracers, such as $\left[{ }^{18} \mathrm{~F}\right]$-fluoromisonidazole (FMISO) [18, 42, 50]. Nevertheless, a recent study showed that due to the extremely complex underlying tumor microstructure, hypoxia imaging using FMISO PET is complex [34]. Since, hybrid PET/MRI allows visualization of different pathophysiological parameters, multi-parametric imaging of the tumor hypoxia seems to be a promising field of research [15, 49]. Two studies investigated the potential of different MR imaging techniques in combination with FDG and FMISO PET imaging in HNC [12, 24]. They concluded that the use of functional MRI sequences in addition to FDG and FMISO PET was of independent, additive value in outcome prognosis.

\section{Technical challenges}

Despite the multiple benefits that integrated PET/MRI offers for RT improvement, there are a number of technical challenges that need to be overcome before PET/MRI data can be properly integrated into RT treatment planning processes.

A major issue for the integration of combined PET/MRI into RT treatment planning is the patient positioning. For RT application, patients have to be positioned in a reproducible way throughout the whole treatment. Therefore, positioning aids such as vacuum mattresses and thermoplastic mask systems are routinely used. Patient positioning, a major prerequisite for accurate integration of multiparametric image data into the treatment planning process, is not yet possible in the integrated PET/MRI system. The development of tools for patient positioning in the PET/ MRI system presents a series of challenges, as other coil a total dose of 60 Gy to the PTV is shown in (c). Regions of interest: PTV (red), brain stem (dark green), brain (light blue) (color figure online)

systems have to be considered if patients are positioned in masks. Furthermore, all devices inserted into the PET/MRI field of view will cause attenuation of the PET signal, which has to be handled in a robust and reproducible manner $[32,48]$.

Modern high-precision RT delivery has improved, achieving a geometric accuracy of just a few millimeters. As a consequence, imaging data that is integrated into the planning process also has to be of high spatial resolution, ideally isotropic. MR images are, under certain conditions, subject to geometric distortions, which might introduce geometric errors affecting tumor delineation as well as dose painting on a voxel basis.

Quantitative PET and MRI is a major issue for the integration of functional imaging into RT planning. Quantification errors will have a direct impact on target volume delineation and even more on dose painting by numbers, where functional data are directly used to shape the dose distribution. PET image quantification is, for example, affected by MR-AC [22]. Whereas, a number of studies and recommendations on quantification issues exist for PET imaging [7, 27], similar investigations for MRI are still lacking.

\section{Conclusions}

Combined PET/MRI offers a unique possibility to examine multiple pathophysiological tumor parameters simultaneously. As a consequence, the integration of PET/MRI data may improve RT treatment planning in terms of more precise target volume delineation, but might also provide a powerful basis for dose painting. Additional potential of PET/MRI for RT lies in follow-up examinations and response assessment. 
Fig. 3 Multi-parametric PET/ MRI of a patient with prostate cancer: Combined $\left[{ }^{11} \mathrm{C}\right]$-choline (CHO) PET/MRI (a), T2weighted TSE MRI (b), ADC map derived from DW MRI (c), $\mathrm{K}_{\mathrm{ep}}$ map as a result of DCE-MRI (d) and dose painting IMRT plan, where a sub-region, on CHO PET, DW- and DCE-MRI, suggesting the presence of the main tumor focus (arrow) was prescribed an escalated dose of 90 Gy instead of 74 Gy, which was prescribed to the whole PTV (e). Regions of interest: PTV (orange), rectum (purple) (color figure online)
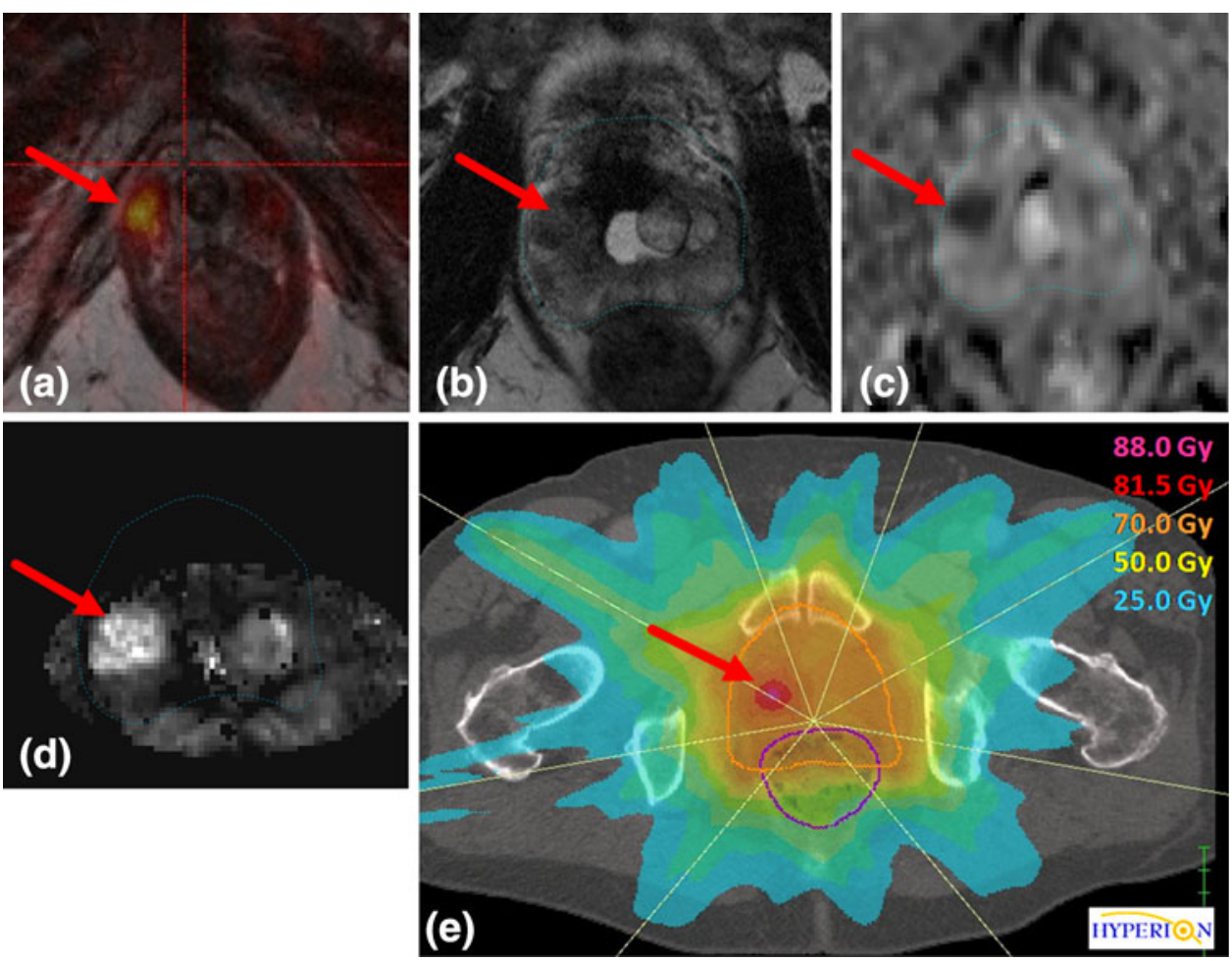

Due to its intrinsic capacity for multi-parametric imaging, hybrid PET/MRI may be a major step towards individualized and personalized $\mathrm{RT}$ in the near future.

Acknowledgments We thank Dr Nina Schwenzer and Dr Holger Schmidt for PET/MRI management and image acquisition. We thank Prof. Dr Markus Alber for providing us with a research version of the RT treatment planning software Hyperion. DT is financially supported by the Ministry of Science, Research and the Arts BadenWürttemberg and the European Social Fund. This work was further supported by fortune grant no. 1945-0-0 of the University Tübingen and by the German Research Foundation, grant no. AL 877/1-3.

\section{Conflict of interest None}

\section{References}

1. Aerts HJ, van Baardwijk AA, Petit SF, Offermann C, Loon J, Houben R, Dingemans AM, Wanders R, Boersma L, Borger J, Bootsma G, Geraedts W, Pitz C, Simons J, Wouters BG, Oellers M, Lambin P, Bosmans G, Dekker AL, De Ruysscher D (2009) Identification of residual metabolic-active areas within individual NSCLC tumours using a pre-radiotherapy (18) Fluorodeoxyglucose-PET-CT scan. Radiother Oncol 91:386-392

2. Aerts HJ, Bussink J, Oyen WJ, van Elmpt W, Folgering AM, Emans D, Velders M, Lambin P, De Ruysscher D (2012) Identification of residual metabolic-active areas within NSCLC tumours using a pre-radiotherapy FDG-PET-CT scan: a prospective validation. Lung Cancer 75:73-76

3. Alber M, Paulsen F, Eschmann SM, Machulla HJ (2003) On biologically conformal boost dose optimization. Phys Med Biol 48:N31-N35
4. Astner ST, Dobrei-Ciuchendea M, Essler M, Bundschuh RA, Sai H, Schwaiger M, Molls M, Weber WA, Grosu AL (2008) Effect of 11C-methionine-positron emission tomography on gross tumor volume delineation in stereotactic radiotherapy of skull base meningiomas. Int J Radiat Oncol Biol Phys 72:1161-1167

5. Bentzen SM (2005) Theragnostic imaging for radiation oncology: dose-painting by numbers. Lancet Oncol 6:112-117

6. Berker Y, Franke J, Salomon A, Palmowski M, Donker HC, Temur Y, Mottaghy FM, Kuhl C, Izquierdo-Garcia D, Fayad ZA, Kiessling F, Schulz V (2012) MRI-based attenuation correction for hybrid PET/MRI systems: a 4-class tissue segmentation technique using a combined ultrashort-echo-time/Dixon MRI sequence. J Nucl Med 53:796-804

7. Boellaard R, O'Doherty MJ, Weber WA et al (2010) FDG PET and PET/CT: EANM procedure guidelines for tumour PET imaging: version 1.0. Eur J Nucl Med Mol Imaging 37:181-200

8. Boss A, Bisdas S, Kolb A, Hofmann M, Ernemann U, Claussen CD, Pfannenberg C, Pichler BJ, Reimold M, Stegger L (2010) Hybrid PET/MRI of intracranial masses: initial experiences and comparison to PET/CT. J Nucl Med 51:1198-1205

9. Brix G, Griebel J, Kiessling F, Wenz F (2010) Tracer kinetic modelling of tumour angiogenesis based on dynamic contrastenhanced CT and MRI measurements. Eur J Nucl Med Mol Imaging 37 Suppl 1:S30-S51

10. Delso G, Martinez-Möller A, Bundschuh RA, Ladebeck R, Candidus Y, Faul D, Ziegler SI (2010) Evaluation of the attenuation properties of MR equipment for its use in a whole-body PET/MR scanner. Phys Med Biol 55:4361-4374

11. Delso G, Fürst S, Jakoby B, Ladebeck R, Ganter C, Nekolla SG, Schwaiger M (2012) Performance measurements of the Siemens mMR integrated whole-body PET/MR scanner. J Nucl Med 52:1914-22. Erratum in: J Nucl Med 53:507

12. Dirix P, Vandecaveye V, De Keyzer F, Stroobants S, Hermans R, Nuyts S (2009) Dose painting in radiotherapy for head and neck squamous cell carcinoma: value of repeated functional imaging with 18F-FDG PET, 18F-fluoromisonidazole PET, diffusion-weighted 
MRI, and dynamic contrast-enhanced MRI. J Nucl Med 50:1020 1027

13. Drzezga A, Souvatzoglou M, Eiber M, Beer AJ, Fürst S, Martinez-Möller A, Nekolla SG, Ziegler S, Ganter C, Rummeny EJ, Schwaiger M (2012) First clinical experience with integrated whole-body PET/MR: comparison to PET/CT in patients with oncologic diagnoses. J Nucl Med 53:845-855

14. Duprez F, De Neve W, De Gersem W, Coghe M, Madani I (2011) Adaptive dose painting by numbers for head-and-neck cancer. Int J Radiat Oncol Biol Phys 80:1045-1055

15. Gaertner FC, Souvatzoglou M, Brix G, Beer AJ (2012) Imaging of hypoxia using PET and MRI. Curr Pharm Biotechnol 13:552-570

16. Gehler B, Paulsen F, Öksüz MÖ et al (2009) [68 Ga]-DOTATOC-PET/CT for meningioma IMRT treatment planning. Radiat Oncol 4:56

17. Groenendaal G, Borren A, Moman MR, Monninkhof E, van Diest PJ, Philippens ME, van Vulpen M, van der Heide UA (2012) Pathologic validation of a model based on diffusion-weighted imaging and dynamic contrast-enhanced magnetic resonance imaging for tumor delineation in the prostate peripheral zone. Int J Radiat Oncol Biol Phys 82: e537-e44

18. Hendrickson K, Phillips M, Smith W, Peterson L, Krohn K, Rajendran J (2011) Hypoxia imaging with [F-18] FMISO-PET in head and neck cancer: potential for guiding intensity modulated radiation therapy in overcoming hypoxia-induced treatment resistance. Radiother Oncol 101:369-375

19. Herzog H, Van Den Hoff J (2012) Combined PET/MR systems: an overview and comparison of currently available options. Q J Nucl Med Mol Imaging 56:247-267

20. Hofmann M, Steinke F, Scheel V, Charpiat G, Farquhar J, Aschoff P, Brady M, Schölkopf B, Pichler BJ (2008) MRI-based attenuation correction for PET/MRI: a novel approach combining pattern recognition and atlas registration. J NuclMed 49:1875-1883

21. Hofmann M, Pichler B, Schölkopf B, Beyer T (2009) Towards quantitative PET/MRI: a review of MR-based attenuation correction techniques. Eur J Nucl Med Mol Imaging 36(Suppl 1):S93S104

22. Hofmann M, Bezrukov I, Mantlik F, Aschoff P, Steinke F, Beyer T, Pichler BJ, Schölkopf B (2011) MRI-based attenuation correction for whole-body PET/MRI: quantitative evaluation of segmentationand atlas-based methods. J Nucl Med 52:1392-1399

23. Jakoby BW, Bercier Y, Conti M, Casey ME, Bendriem B, Townsend DW (2011) Physical and clinical performance of the mCT time-of-flight PET/CT scanner. Phys Med Biol 56:2375-2389

24. Jansen JFA, Schöder H, Lee NY, Wang Y, Pfister DG, Fury MG, Stambuk HE, Humm JL, Koutcher JA, Shukla-Dave A (2010) Noninvasive assessment of tumour microenvironment using dynamic contrast-enhanced magnetic resonance imaging and $18 \mathrm{~F}$-fluoromisonidazole positron emission tomography imaging in neck nodal metastases. Int $\mathrm{J}$ Radiat Oncol Biol Phys 77:1403-1410

25. Judenhofer MS, Wehrl HF, Newport DF, Catana C, Siegel SB, Becker M, Thielscher A, Kneilling M, Lichy MP, Eichner M, Klingel K, Reischl G, Widmaier S, Röcken M, Nutt RE, Machulla HJ, Uludag K, Cherry SR, Claussen CD, Pichler BJ (2008) Simultaneous PET-MRI: a new approach for functional and morphological imaging. NatMed 14:459-465

26. Kinahan PE, Hasegawa BH, Beyer T (2003) X-ray-based attenuation correction for positron emission tomography/computed tomography scanners. Semin Nucl Med 33:166-179

27. Lee JA (2010) Segmentation of positron emission tomography images: some recommendations for target delineation in radiation oncology. Radiother Oncol 96:302-307

28. Ling CC, Humm J, Larson Amols H et al (2000) Towards multidimensional radiotherapy (MD-CRT): biological imaging and biological conformality. Int $\mathrm{J}$ Radiol Oncol Biol Phys 47:551-560

29. Lips IM, van der Heide UA, Haustermans K, van Lin EN, Pos F, Franken SP, Kotte AN, van Gils CH, van Vulpen M (2011) Single blind randomized phase III trial to investigate the benefit of a focal lesion ablative microboost in prostate cancer (FLAMEtrial): study protocol for a randomized controlled trial. Trials $12: 255$

30. Lois C, Bezrukov I, Schmidt H, Schwenzer N, Werner MK, Kupferschläger J, Beyer T (2012) Effect of MR contrast agents on quantitative accuracy of PET in combined whole-body PET/ MR imaging. Eur J Nucl Med Mol Imaging 39:1756-1766

31. Malinen E, Søvik A, Hristov D, Bruland ØS, Olsen DR (2006) Adapting radiotherapy to hypoxic tumours. Phys Med Biol 51:4903-4921

32. Mantlik F, Hofmann M, Werner MK, Sauter A, Kupferschläger J, Schölkopf B, Pichler BJ, Beyer T (2011) The effect of patient positioning aids on PET quantification in PET/MR imaging. Eur $\mathrm{J}$ Nucl Med Mol Imaging 38:920-929

33. Martinez-Möller A, Souvatzoglou M, Delso G, Bundschuh RA, Chefd'hotel C, Ziegler SI, Navab N, Schwaiger M, Nekolla SG (2009) Tissue classification as a potential approach for attenuation correction in whole-body PET/MRI: evaluation with PET/CT data. J Nucl Med 50:520-526

34. Mönnich D, Troost EG, Kaanders JH, Oyen WJ, Alber M, Thorwarth D (2011) Modelling and simulation of [18F]fluoromisonidazole dynamics based on histology-derived microvessel maps. Phys Med Biol 56:2045-2057

35. Muzik J, Soukup M, Alber M (2008) Comparison of fixed-beam IMRT, helical tomotherapy, and IMPT for selected cases. Med Phys 35:1580-1592

36. Nestle U, Weber W, Hentschel M, Grosu AL (2009) Biological imaging in radiation therapy: role of positron emission tomography. Phys Med Biol 54:R1-R25

37. Oelfke U, Bortfeld T (2003) Optimization of physical dose distributions with hadron beams: comparing photon IMRT with IMPT. Technol Cancer Res Treat 2:401-412

38. Otto K (2008) Volumetric modulated arc therapy: IMRT in a single gantry arc. Med Phys 35:310-317

39. Padhani AR, Liu G, Koh DM, Chenevert TL, Thoeny HC, Takahara T, Dzik-Jurasz A, Ross BD, Van Cauteren M, Collins D, Hammoud DA, Rustin GJ, Taouli B, Choyke PL (2009) Diffusion-weighted magnetic resonance imaging as a cancer biomarker: consensus and recommendations. Neoplasia 11:102-125

40. Pichler BJ, Kolb A, Nägele T, Schlemmer HP (2010) PET/MRI: paving the way for the next generation of clinical multimodality imaging applications. J Nucl Med 51:333-336

41. Piert M, Park H, Khan A, Siddiqui J, Hussain H, Chenevert T, Wood D, Johnson T, Shah RB, Meyer C (2009) Detection of aggressive primary prostate cancer with $11 \mathrm{C}$-choline PET/CT using multimodality fusion techniques. J Nucl Med 50:15851593

42. Rischin D, Hicks RJ, Fisher R et al (2006) Trans-Tasman radiation oncology group study 98.02. Prognostic significance of $[18 \mathrm{~F}]-$ misonidazole positron emission tomography-detected tumor hypoxia in patients with advanced head and neck cancer randomly assigned to chemoradiation with or without tirapazamine: a substudy of Trans-Tasman radiation oncology group study 98.02. J Clin Oncol 24:2098-2104

43. Rit S, Nijkamp J, van Herk M, Sonke JJ (2011) Comparative study of respiratory motion correction techniques in cone-beam computed tomography. Radiother Oncol 100:356-359

44. Røe K, Kakar M, Seierstad T, Ree AH, Olsen DR (2011) Early prediction of response to radiotherapy and androgen-deprivation therapy in prostate cancer by repeated functional MRI: a preclinical study. Radiat Oncol 6:65 
45. Schwenzer NF, Schraml C, Müller M, Brendle C, Sauter A, Spengler W, Pfannenberg AC, Claussen CD, Schmidt H (2012) Pulmonary lesion assessment: comparison of whole-body hybrid $\mathrm{MR} / \mathrm{PET}$ and PET/CT imaging—pilot study. Radiology 264:551-558

46. Schwenzer NF, Stegger L, Bisdas S, Schraml C, Kolb A, Boss A, Müller M, Reimold M, Ernemann U, Claussen CD, Pfannenberg C, Schmidt $\mathrm{H}$ et al (2012) Simultaneous PET/MR imaging in a human brain PET/MR system in 50 patients-current state of image quality. Eur J Radio 181(11):3472-3478

47. Tang C, Murphy JD, Khong B et al (2012) Validation that metabolic tumor volume predicts outcome in head-and-neck cancer. Int J Radiat Oncol Biol Phys 83:1514-1520

48. Tellmann L, Quick HH, Bockisch A, Herzog H, Beyer T (2011) The effect of MR surface coils on PET quantification in wholebody PET/MR: results from a pseudo-PET/MR phantom study. Med Phys 38:2795-2805

49. Thorwarth D, Alber M (2010) Implementation of hypoxia imaging into treatment planning and delivery. Radiother Oncol 197:172-175

50. Thorwarth D, Eschmann SM, Scheiderbauer J, Paulsen F, Alber M (2006) Kinetic analysis of dynamic 18F-fluoromisonidazole PET correlates with radiation treatment outcome in head-andneck cancer. BMC Cancer 5:152

51. Thorwarth D, Eschmann SM, Paulsen F, Alber M (2007) Hypoxia dose painting by numbers: a planning study. Int $\mathrm{J}$ Radiat Oncol Biol Phys 68:291-300
52. Thorwarth D, Henke G, Müller AC, Reimold M, Beyer T, Boss A, Kolb A, Pichler B, Pfannenberg C (2011) Simultaneous 68 Ga-DOTATOC-PET/MRI for IMRT treatment planning for meningioma: first experience. Int J Radiat Oncol Biol Phys $81: 277-283$

53. Turkbey B, Pinto PA, Mani H et al (2010) Prostate cancer: value of multiparametric MR imaging at $3 \mathrm{~T}$ for detection-histopathologic correlation. Radiology 255:89-99

54. Van den Bergh L, Koole M, Isebaert S, Joniau S, Deroose CM, Oyen R, Lerut E, Budiharto T, Mottaghy F, Bormans G, Van Poppel H, Haustermans K (2012) Is there an additional value of ${ }^{11} \mathrm{C}$-choline PET-CT to T2-weighted MRI images in the localization of intraprostatic tumor nodules? Int J Radiat Oncol Biol Phys 83:1486-1492

55. van der Heide UA, Houweling AC, Groenendaal G, Beets-Tan RG, Lambin P (2012) Functional MRI for radiotherapy dose painting. Magn Reson Imaging 30:1216-1223

56. Veit-Haibach P, Kuhn FP, Wiesinger F, Delso G, von Schulthess G (2012) PET-MR imaging using a tri-modality PET/CT-MR system with a dedicated shuttle in clinical routine. Magn Reson Mater Phy. doi: 10.1007/s10334-012-0344-5

57. Wehrl HF, Sauter AW, Judenhofer MS, Pichler BJ (2010) Combined PET/MR imaging-technology and applications. Technol Cancer Res Treat 9:5-20

58. Werner MK, Schmidt H, Schwenzer NF (2012) MR/PET: a new challenge in hybrid imaging. AJR Am J Roentgenol 199:272-277 